\title{
Comprensión De Una Tabla Estadística Por Estudiantes Universitarios En México Y Chile
}

\author{
Understanding Of A Statistical Table By University Students In Mexico And Chile
}

\author{
Jaime I. García-García* \\ Isaac Alejandro Imilpán Rivera** \\ Elizabeth-H. Arredondo ${ }^{* * *}$ \\ Nicolás Alonso Fernández Coronado ${ }^{* * * *}$ \\ Universidad de Los Lagos (ULagos)
}

\begin{abstract}
Resumen
Uno de los principales desafíos de la Educación Estadística consiste en dotar a los estudiantes de la capacidad de comprender información presentada en tablas o gráficas para concebir su entorno, para establecer conclusiones y tomar decisiones. En la presente investigación, de tipo cualitativaexploratoria, se analiza y compara el nivel de comprensión de una tabla estadística por estudiantes universitarios de México y Chile, con miras a identificar diferencias. Las respuestas se analizaron bajo una jerarquía condensada a partir de los niveles de lectura de Curcio y la jerarquía de Aoyama. En general, ambos grupos de estudiantes alcanzan el nivel 2 (comparativo) al realizar una comparación con los datos; sin embargo, en este mismo nivel se presenta un mayor número de falencias en la identificación de aumentos/decrementos y/o cálculos aritméticos con los datos.
\end{abstract}

Palabras clave: Niveles de comprensión, Tabla estadística, Cultura estadística, Universidad.

\begin{abstract}
One of the main challenges of Statistical Education is to provide students with the ability to understand information presented in tables or graphs to conceive the environment that surrounds them draw conclusions and make decisions. In the present research, of a qualitative-exploratory type, the level of understanding of a statistical table by university students from Mexico and Chile, is analyzed and compared with a view to identifying differences. The answers were analyzed under a condensed hierarchy based on Curcio's reading levels and the Aoyama's hierarchy. In general, both groups of students reach level 2 (comparative) by making a comparison with the data; however, in this same level there is a greater number of mistakes in the identification of increases/decreases and/or arithmetic calculations done with the data.
\end{abstract}

Keywords: Levels of understanding, Statistical table, Literacy statistical, University.

\footnotetext{
${ }^{*}$ Doctor en Ciencias, Especialidad en Matemática Educativa, por el Cinvestav-IPN. Académico de los Postgrados en Educación Matemática de la ULagos, Osorno, Chile. E-mail: jaime.garcia@ulagos.cl.

** Estudiante de Pedagogía en Educación Media en Matemática y Computación de la Universidad de Los Lagos, Osorno, Chile. E-mail: isaacalejandro.imilpan@alumnos.ulagos.cl.

*** Doctora en Ciencias, Especialidad en Matemática Educativa, por el Cinvestav-IPN. Académica de los Postgrados en Educación Matemática de la ULagos, Osorno, Chile. E-mail: elizabeth.hernandez@ulagos.cl.

**** Estudiante de Pedagogía en Educación Media en Matemática y Computación de la Universidad de Los Lagos, Osorno, Chile. E-mail: nicolasalonso.fernandez@alumnos.ulagos.cl.
} 


\section{Introducción}

La Estadística ha ido tomando una creciente relevancia en los últimos años, ya que entrega las herramientas necesarias para desarrollar la capacidad de comprender la información contenida en tablas o gráficas que frecuentemente presentan diversos medios de comunicación (Arteaga, Batanero, Cañadas y Contreras, 2011; Beltrão, 2012; Cabral dos Santos y Selva, 2011; Díaz-Levicoy, Batanero, Arteaga y López-Martín, 2015; Silva y Guimarães, 2013).

Estrella y Olfos (2012) señalan la comprensión gráfica como la lectura e interpretación de este tipo de representaciones; bajo esta perspectiva, consideramos la comprensión de tablas estadísticas como la capacidad de leerlas e interpretarlas, que le permite al estudiante establecer conclusiones, entender su entorno y tomar decisiones adecuadas.

Esto último es fundamental dentro de las diversas áreas de formación académica de estudiantes universitarios: ciencias exactas, sociales, de la salud, de la ingeniería, entre otras. Autores como Eudave (2009), consideran la capacidad de leer y entender datos estadísticos como una necesidad educativa y social relativamente nueva. En este sentido, Ridgway, Nicholson y McCusker (2008) señalan la necesidad de que los ciudadanos sean estadísticamente cultos.

Las tablas y gráficas estadísticas, así como su comprensión, forman parte importante de la cultura estadística (Arteaga, Batanero, Cañadas, Contreras, 2011; Del Pino y Estrella, 2012; Gal, 2002). Esta cultura consiste en interpretar y evaluar críticamente información estadística; así como también, en formular y comunicar opiniones respecto a dicha información (Gal y Murray, 2011).

Una tabla estadística, según Estrella (2014), es una forma de representación rectangular que permiten mostrar datos correspondientes a una o más variables de manera clara y resumida, visualizar el comportamiento de los datos y facilitar la comprensión de la información.

Ante todo esto, nacen dos interrogantes: ¿qué nivel de comprensión sobre tablas estadísticas alcanzan los estudiantes universitarios?, ¿qué diferencias pueden presentarse en esta comprensión, sujeta a contextos geográficos y socioculturales diferentes, por estudiantes de México y Chile? Para dar respuesta a estas preguntas, en esta investigación se analiza el nivel de comprensión de una tabla estadística por dos grupos de estudiantes universitarios, uno de México y otro de Chile. 


\section{Algunos Estudios Sobre Tablas Estadísticas}

Hasta el momento, los estudios sobre tablas estadísticas han tenido como objetivo analizar la comprensión de los estudiantes, en diversos niveles educativos, y su presencia en los libros de texto (ver Tabla 1). Enseguida se describen las investigaciones relacionadas con el primer objetivo con estudiantes de secundaria, universitarios y profesores en servicio; las cuales nos sirven para situar nuestro trabajo.

Tabla 1: Clasificación de algunas investigaciones sobre tablas estadísticas

\begin{tabular}{|c|c|c|c|c|c|}
\hline Libros de texto & $\begin{array}{l}\text { Educación } \\
\text { Infantil }\end{array}$ & $\begin{array}{l}\text { Educación } \\
\text { Primaria }\end{array}$ & $\begin{array}{l}\text { Educación } \\
\text { Secundaria }\end{array}$ & Universidad & $\begin{array}{c}\text { Profesores en } \\
\text { Servicio }\end{array}$ \\
\hline $\begin{array}{l}\text { Amorim y Silva } \\
(2016) \\
\text { Díaz-Levicoy, } \\
\text { Morales y } \\
\text { López-Martín } \\
\text { (2015) Bivar y } \\
\text { Selva (2011) } \\
\text { Guimarães, } \\
\text { Gitirana, } \\
\text { Cavalcanti y } \\
\text { Marques (2008) }\end{array}$ & $\begin{array}{l}\text { Fuentes, } \\
\text { Arteaga y } \\
\text { Batanero } \\
(2014)\end{array}$ & $\begin{array}{l}\text { Sepúlveda, } \\
\text { Díaz-Levicoy } \\
\text { y Jara (2018) } \\
\text { Estrella y } \\
\text { Olfos (2015) } \\
\text { Ochoa (2015) } \\
\text { Espinoza } \\
\text { (2015) } \\
\text { Pereira y Conti } \\
\text { (2012) } \\
\text { Gabucio, } \\
\text { Martí, } \\
\text { Enfedaque, } \\
\text { Gilabert y } \\
\text { Konstantinidou } \\
\text { (2010) } \\
\text { Pagan, Leite, } \\
\text { Magina y } \\
\text { Cazorla (2008) }\end{array}$ & $\begin{array}{l}\text { Carmona y } \\
\text { Cruz (2016) } \\
\text { Eudave (2009) }\end{array}$ & $\begin{array}{l}\text { Arredondo, } \\
\text { García-García } \\
\text { y López (2019) } \\
\text { Díaz-Levicoy, } \\
\text { Sepúlveda, } \\
\text { Vásquez y } \\
\text { Opazo (2016) }\end{array}$ & $\begin{array}{l}\text { Rodríguez y } \\
\text { Sandoval (2012) }\end{array}$ \\
\hline
\end{tabular}

Fuente: Elaborada por los autores

Eudave (2009) realiza una investigación con 28 estudiantes, de distintas edades, que cursaban la primaria o secundaria en alguna de las modalidades de educación para adultos que se imparte en México; destacando que únicamente 5 de ellos poseen los tres niveles de comprensión contemplados por Curcio, ya que pudieron realizar una lectura completa (incluyendo predicciones) y adecuada de la tabla de frecuencias y de la gráfica de líneas. Por su parte, Díaz-Levicoy, Sepúlveda, Vásquez y Opazo (2016) realizan un estudio sobre los niveles de lectura de tablas estadísticas que alcanzan 121 futuras profesoras de educación infantil; señalan que la mayoría de las respuestas se ubican en los niveles leer los datos y leer dentro de los datos, asociados a la lectura literal de información y procesos matemáticos sencillos, respectivamente.

En Carmona y Cruz (2016) se reportan las características que favorecen la comprensión de la información contenida en tablas y gráficas estadísticas de 55 estudiantes del grado 
séptimo; observan que en su mayoría, las respuestas se concentran en los primeros niveles de la jerarquía de Aoyama. Arredondo, García-García y López (2019) realizan un análisis de las interpretaciones de 36 estudiantes universitarios de una tabla y un gráfico de líneas, encontrando que la mayor proporción de los participantes alcanza un nivel intermedio de lectura, leer dentro de los datos; mientras que pocos jóvenes alcanzaron niveles superiores, leer más allá de los datos y leer detrás de los datos, respectivamente. Rodríguez y Sandoval (2012) analizan la lectura y construcción de gráficas y tablas estadísticas de 47 profesores chilenos en ejercicio y 44 en formación; evidenciando que, en general, los participantes se ubican en el nivel 1 de Curcio, es decir, presentan habilidades básicas o iniciales asociadas al análisis de los datos.

A partir de esta revisión de literatura, se identifica que los estudios sobre tablas estadísticas con estudiantes son escasos en Educación Media y Superior. Bajo dicha perspectiva, esta investigación se presenta como novedosa dentro del área de la Educación Estadística.

\section{Referentes Teóricos}

Una manera de describir la comprensión de tablas estadísticas, es analizando y caracterizando las respuestas de los estudiantes a la tarea de leer e interpretar información presente en este tipo de representaciones, a través un modelo jerárquico. Curcio (1989) y Friel, Curcio y Bright (2001) establecen cuatro niveles de lectura de gráficas que permiten describir las diferencias en las habilidades en estas representaciones estadísticas:

- Nivel 1. Leer los datos. Es la lectura literal de la información presentada en la gráfica, sin realizar su interpretación ni cálculos adicionales. Por ejemplo, leer la frecuencia asociada a un valor de la variable, el título o la fuente, entre otros.

- Nivel 2. Leer dentro de los datos. Es la integración de la información presente en la gráfica, que no está representada explícitamente; esto implica la comparación de datos o la realización de cálculos matemáticos con ellos. Por ejemplo, identificar la moda de la variable al comparar todas las frecuencias para encontrar la mayor.

- Nivel 3. Leer más allá de los datos. Corresponde a la realización de predicciones o inferencias a partir de los datos sobre información que no se presenta directamente en la gráfica. Por ejemplo, predecir tendencias sobre el comportamiento de los datos.

- Nivel 4. Leer detrás de los datos. Implica la valoración crítica del uso de la 
gráfica, la recogida y organización de los datos, su validez y fiabilidad; así como la integración de la información con el contexto para extraer conclusiones. Por ejemplo, cuestionarse sobre la calidad de los datos y la forma de recolección.

Estos niveles han sido considerados como un modelo jerárquico para evaluar y caracterizar la lectura de gráficas estadísticas, no obstante, puede aplicarse para la lectura de tablas (Batanero, 2001), tal es el caso de los estudios realizados por Eudave (2009) y Arredondo, et al. (2019).

En nuestra investigación surgió la necesidad de analizar a profundidad la respuesta, del estudiante, enfocada en la valoración crítica de la información, por ello nos apoyamos en la jerarquía propuesta por Aoyama (2007), la cual establece cinco niveles de interpretación de gráficas:

- Nivel 1. Idiosincrático. Los estudiantes no leen valores o tendencias en las gráficas, proporcionan valores incorrectos o dejan de contestar la pregunta. Por lo general, sus respuestas se basan en su experiencia individual o en perspectivas personales.

- Nivel 2. Lectura básica. Los estudiantes leen valores y tendencias en las gráficas, pero no pueden explicar los significados contextuales de las tendencias o características que ven, ni contextualizar los eventos presentados.

- Nivel 3. Racional/literal. Los estudiantes leen valores y tendencias particulares, explican los significados contextuales literalmente en términos de los rasgos mostrados en la gráfica, pero no pueden sugerir ninguna interpretación alternativa.

- Nivel 4. Crítico. Los estudiantes leen las gráficas y comprenden las variables contextuales presentadas; además de evaluar la fiabilidad del significado contextual descrito en la gráfica y cuestionar la información presentada.

- Nivel 5. Elaboración de hipótesis y modelos. Los estudiantes leen las gráficas, aceptan y evalúan alguna información presentada, al formar sus propias hipótesis o modelos explicativos.

La interrelación entre ambas jerarquías permitió analizar la comprensión respecto a los elementos que recuperan los estudiantes cuando presentan el nivel leer detrás de los datos; esto llevo a establecer los tres niveles superiores de Aoyama (racional/literal, crítico y elaboración de hipótesis y modelos) como subcategorías del nivel 4 de Curcio. La consideración de estos modelos jerárquicos nace de la potencialidad que diversos autores notan en ellas (e.g., Batanero, 
Díaz-Levicoy y Arteaga, 2018; Carmona y Cruz, 2016; Gea, Arteaga y Cañadas, 2017).

\section{Metodología}

La metodología de la investigación se enmarcó bajo un enfoque cualitativo, de tipo exploratorio-descriptivo, ya que se analiza el nivel de comprensión presente en las respuestas de estudiantes universitarios a una tarea que involucra leer e interpretar una tabla estadística.

\subsection{Participantes}

En esta investigación participaron dos grupos de estudiantes universitarios. El Grupo 1 estaba formado por 36 estudiantes primer semestre de la Licenciatura en Matemáticas de la Universidad Autónoma de Guerrero, México; mientras que el Grupo 2 estaba formado por 35 alumnos de primer semestre de la carrera de Pedagogía en Matemáticas y Computación de la Universidad de Los Lagos, Chile. Los participantes de ambos grupos, cuyas edades oscilaban entre los 18 y 34 años, fueron seleccionados mediante un muestro no probabilístico por conveniencia, y no recibieron información alguna sobre el propósito del estudio. El profesor titular de cada grupo colaboró con la aplicación de la tarea.

\subsection{Tareas Y Su Aplicación}

Dos tareas de comprensión de información representada en tablas estadísticas; específicamente se les solicitó a los estudiantes leer, interpretar y comparar datos, observar tendencias, predecir, generar conclusiones y realizar críticas, en general, redactar varios enunciados acerca de la información. La tabla de la tarea A (ver Figura 1) muestra el porcentaje de hogares con televisión y con televisión de paga en México durante el periodo de 2013 a 2016; mientras que la tabla de la tarea B (ver Figura 2) presenta información acerca de la evolución de titulados de carreras de pregrado por género en la Universidad de Los Lagos de 2012 a 2016. Cabe aclarar que las tareas propuestas podrían ser consideradas de diferente nivel de complejidad por el tipo de frecuencia (relativa en porcentaje y absoluta); sin embargo, asumimos que esto no afecta el nivel de comprensión que alcanza el estudiante, ya que con la información dada puede efectuar comparaciones, predicciones y/o críticas. 
PORCENTAJE DE HOGARES CON TELEVISIÓN Y CON TELEVISIÓN DE PAGA EN MÉXICO

\begin{tabular}{|c|c|c|}
\hline Año & Con televisión & Con televisión de paga \\
\hline 2013 & $94.9 \%$ & $36.7 \%$ \\
\hline 2014 & $94.9 \%$ & $38.1 \%$ \\
\hline 2015 & $93.5 \%$ & $43.7 \%$ \\
\hline 2016 & $93.1 \%$ & $52.1 \%$ \\
\hline
\end{tabular}

Figura 1: Tabla de la tarea A

Fuente: Elaborada por los autores

EVOLUCIÓN DE TITULADOS CARRERAS DE PREGRADO POR GÉNERO

\begin{tabular}{|l|c|c|c|c|c|}
\hline Género & 2012 & 2013 & 2014 & 2015 & 2016 \\
\hline Femenino & 787 & 902 & 975 & 1020 & 1067 \\
\hline Masculino & 699 & 724 & 778 & 813 & 767 \\
\hline Total general & 1486 & 1626 & 1753 & 1833 & 1834 \\
\hline
\end{tabular}

Fuente: Anuario Estadístico Institucional 2016, Universidad de Los Lagos

Figura 2: Tabla de la tarea B

Fuente: Universidad de Los Lagos (2016, p. 32)

La tarea de leer e interpretar la información representada en la tabla se consideró de manera abierta, es decir, sin preguntas. Esta consideración se debió a que el estudiante comúnmente se enfrenta a este proceso de comprensión de tablas estadísticas, presentadas por diversos medios de comunicación, sin que se le guíe.

La tarea A se aplicó en una sesión a los 36 estudiantes mexicanos; mientras que la tarea B, también en una sesión, a los 35 estudiantes chilenos. Cada sesión duró aproximadamente 20 minutos. Cabe mencionar que la información elegida y representada en las tablas estadísticas es real y se acerca al contexto en el que se desenvuelve el estudiante. La tabla A es de elaboración propia, mientras que la tabla B fue tomada del Anuario Estadístico Institucional de la Universidad de Los Lagos (2016).

\subsection{Análisis De Datos}

Las respuestas de los estudiantes se analizan y clasifican, mediante triangulación de expertos, de acuerdo a la comprensión de su información. Para hacerlo se han tomado como guía los modelos jerárquicos descritos en los referentes teóricos, condensándolos en una sola 
jerarquía (ver Tabla 2).

Tabla 2: Jerarquía propuesta para el análisis de la comprensión de tablas estadísticas

\begin{tabular}{|c|c|c|c|}
\hline $\begin{array}{c}\text { Nivel de } \\
\text { comprensión }\end{array}$ & $\begin{array}{l}\text { Nivel de } \\
\text { Curcio }\end{array}$ & $\begin{array}{l}\text { Nivel de } \\
\text { Aoyama }\end{array}$ & Descripción \\
\hline $\begin{array}{l}\text { Nivel } 0 . \\
\text { Perspectiva } \\
\text { personal }\end{array}$ & - & $\begin{array}{l}\text { Nivel } 1 . \\
\text { Idiosincrático }\end{array}$ & $\begin{array}{l}\text { La respuesta del estudiante se basa en perspectivas } \\
\text { personales del estudiante. }\end{array}$ \\
\hline $\begin{array}{l}\text { Nivel } 1 . \\
\text { Lectura } \\
\text { literal }\end{array}$ & $\begin{array}{l}\text { Nivel 1. Leer } \\
\text { los datos }\end{array}$ & \multirow{3}{*}{$\begin{array}{l}\text { Nivel } 2 . \\
\text { Lectura básica. }\end{array}$} & $\begin{array}{l}\text { La respuesta del estudiante presenta palabras referentes al } \\
\text { título o a la(s) variable(s) de la tabla; o bien, frecuencias } \\
\text { correspondientes a los valores de la(s) variable(s); sin } \\
\text { realizar interpretaciones de la información. }\end{array}$ \\
\hline $\begin{array}{l}\text { Nivel } 2 . \\
\text { Comparativo }\end{array}$ & $\begin{array}{l}\text { Nivel 2. Leer } \\
\text { dentro de los } \\
\text { datos }\end{array}$ & & $\begin{array}{l}\text { La respuesta del estudiante presenta comparaciones de } \\
\text { datos por columnas, por filas, o bien, de ambas maneras. }\end{array}$ \\
\hline $\begin{array}{l}\text { Nivel } 3 . \\
\text { Predictivo }\end{array}$ & $\begin{array}{l}\text { Nivel 3. Leer } \\
\text { más allá de los } \\
\text { datos }\end{array}$ & & $\begin{array}{l}\text { La respuesta del estudiante presenta predicciones de } \\
\text { tendencias del comportamiento de los datos o de } \\
\text { frecuencias correspondientes a los valores de la(s) } \\
\text { variable(s) de la tabla. }\end{array}$ \\
\hline \multirow{3}{*}{$\begin{array}{l}\text { Nivel } 4 . \\
\text { Integrativo }\end{array}$} & \multirow{3}{*}{$\begin{array}{l}\text { Nivel 4. Leer } \\
\text { detrás de los } \\
\text { datos }\end{array}$} & $\begin{array}{l}\text { Nivel } 3 . \\
\text { Racional/literal }\end{array}$ & $\begin{array}{l}\text { La respuesta del estudiante presenta una valoración crítica } \\
\text { de la información al integrar una conexión con el } \\
\text { contexto, de manera: } \\
\text { a) racional/literal, al explicar significados } \\
\text { contextuales literalmente en términos de los datos } \\
\text { mostrados en la tabla, pero sin cuestionar la } \\
\text { información o sugerir alguna interpretación } \\
\text { alternativa. }\end{array}$ \\
\hline & & $\begin{array}{l}\text { Nivel } 4 . \\
\text { Crítico }\end{array}$ & $\begin{array}{l}\text { b) crítica, al evaluar la fiabilidad de la información } \\
\text { y/o la forma en que se recolecta o presentan los } \\
\text { datos. }\end{array}$ \\
\hline & & $\begin{array}{l}\text { Nivel } 5 . \\
\text { Elaboración de } \\
\text { hipótesis y } \\
\text { modelos }\end{array}$ & $\begin{array}{l}\text { c) hipotética, al aceptar y evaluar alguna } \\
\text { información formando hipótesis explicativas. }\end{array}$ \\
\hline
\end{tabular}

Fuente: Elaborada por los autores

Además, mediante un análisis comparativo de las respuestas, se identifican los incidentes presentes que hicieran referencia a una misma idea; es decir, los aspectos o elementos característicos que los estudiantes consideran en su lectura e interpretación.

\section{Resultados}

En la Tabla 3 se presenta la trascripción de algunas respuestas dadas por los estudiantes a la tarea de leer e interpretar una tabla estadística. Estas se clasifican de acuerdo al nivel de comprensión alcanzado, seguidas de una breve justificación sobre esta clasificación. Las letras en negrita de la primera columna, de izquierda a derecha, resaltan los aspectos o elementos característicos presentes en las respuestas de los estudiantes, que se consideraron para la clasificación; mientras que letras subrayadas de la segunda columna indican el nivel alcanzado. 
Tabla 3: Clasificación de algunas respuestas de acuerdo al nivel de comprensión alcanzado

\begin{tabular}{|c|c|c|}
\hline Tarea, estudiante y respuesta & $\begin{array}{c}\text { Nivel de } \\
\text { comprensión }\end{array}$ & Descripción \\
\hline $\begin{array}{l}\text { Tarea A, estudiante 11: } \\
\text { Desde los años } 2013 \text { al } 2016 \text { disminuyo } \\
\text { cierto porcentaje en hogares con televisión } \\
\text { y aumento el porcentaje de hogares con } \\
\text { televisión de paga. } \\
\text { Del } 2013 \text { al } 2016 \text { hubo un decrecimiento } \\
\text { del } 1.8 \% \text { en hogares con televisión y el } \\
15.4 \% \text { aumento en hogares con televisión } \\
\text { de paga. }\end{array}$ & $\begin{array}{l}\text { Nivel } 1 . \\
\text { Lectura literal } \\
\text { Nivel } 2 . \\
\text { Comparativo }\end{array}$ & $\begin{array}{l}\text { La respuesta del estudiante presenta } \\
\text { palabras ligadas directamente a las } \\
\text { variables, por ejemplo 'hogares con } \\
\text { televisión'; además, exhibe comparaciones } \\
\text { de los datos por columnas, al indicar } \\
\text { expresiones como 'aumento' o } \\
\text { 'disminuyo'. }\end{array}$ \\
\hline $\begin{array}{l}\text { Tarea A, estudiante } 28 \text { : } \\
\text { En el año } 2013 \text { el porcentaje de hogares con } \\
\text { televisión es de } 94.9 \% \text { y con televisión de } \\
\text { paga es de } 36.7 \% \\
\text { Esto representa cada vez más que aumenta } \\
\text { hogares con televisión de paga en México y } \\
\text { hogares con televisión. }\end{array}$ & $\begin{array}{l}\text { Nivel } 1 . \\
\text { Lectura literal } \\
\frac{\text { Nivel } 2 .}{\text { Comparativo }} \\
\text { (Inadecuado) }\end{array}$ & $\begin{array}{l}\text { La respuesta del estudiante presenta } \\
\text { palabras ligadas directamente a las } \\
\text { variables aunada con su porcentaje, por } \\
\text { ejemplo 'porcentaje de hogares con } \\
\text { televisión es de } 94.9 \% \text { '. Además, exhibe } \\
\text { comparaciones de los datos por columnas, } \\
\text { al indicar expresiones como 'cada vez más } \\
\text { que aumenta'. Sin embargo, en esta } \\
\text { comparación presenta una falencia al } \\
\text { indicar que los valores de la variable } \\
\text { 'hogares con televisión' también } \\
\text { aumentan. }\end{array}$ \\
\hline $\begin{array}{l}\text { Tarea A, estudiante } 34 \text { : } \\
\text { Según en los hogares de México ha ido } \\
\text { aumentando el porcentaje en televisiones de } \\
\text { paga y ha disminuido la otra opción que es } \\
\text { solo televisión. } \\
\text { En algún año la televisión de paga ocupará } \\
\text { el 90\% o más en México. } \\
\text { En el } 2013 \text { - } 2014 \text { el porcentaje con } \\
\text { televisión no se vio afectado, siguió igual, } \\
\text { hasta que empezó a decrecer. }\end{array}$ & $\begin{array}{l}\text { Nivel } 1 . \\
\text { Lectura literal } \\
\text { Nivel } 2 \text {. } \\
\text { Comparativo }\end{array}$ & $\begin{array}{l}\text { La respuesta del estudiante presenta } \\
\text { expresiones ligadas directamente a las } \\
\text { variables, por ejemplo 'porcentaje con } \\
\text { televisión'; comparaciones de los datos por } \\
\text { columnas, al indicar palabras como } \\
\text { 'aumentando - disminuido'; y la } \\
\text { predicción de un valor: 'En algún año la } \\
\text { televisión de paga ocupará el } 90 \% \text { o más } \\
\text { en México'. }\end{array}$ \\
\hline $\begin{array}{l}\text { Tarea A, estudiante 9: } \\
\text { En la tabla se puede observar que del año } \\
2013 \text { al } 2016 \text { el porcentaje de hogares con } \\
\text { televisión y con televisión de paga. } \\
\text { Ha disminuido con sólo televisión, por lo } \\
\text { contrario con TV de paga que ha ido en } \\
\text { aumento, tal vez porque la gente ya no } \\
\text { puede comprar televisores porque son } \\
\text { caros y se ven presionados a contratar } \\
\text { T.V. de paga por sus teles viejas. }\end{array}$ & $\begin{array}{l}\text { Nivel } 1 . \\
\text { Lectura literal } \\
\text { Nivel } 2 . \\
\text { Comparativo } \\
\text { Nivel } 4 . \\
\text { Integrativo }\end{array}$ & $\begin{array}{l}\text { La respuesta del estudiante presenta } \\
\text { palabras ligadas directamente a las } \\
\text { variables, por ejemplo 'hogares con } \\
\text { televisión y con televisión de paga'; } \\
\text { comparaciones de los datos por columnas, } \\
\text { al indicar palabras como 'disminuido - } \\
\text { aumento'; y se exhibe una integración con } \\
\text { el contexto, dando hipótesis alternativas } \\
\text { sobre el comportamiento de los datos: 'tal } \\
\text { vez porque la gente ya no puede comprar } \\
\text { televisores porque son caros y se ven } \\
\text { presionados a contratar T.V. de paga por } \\
\text { sus teles viejas'. }\end{array}$ \\
\hline $\begin{array}{l}\text { Tarea } \mathrm{B} \text {, estudiante } 22 \text { : } \\
\text { La información de la evolución de titulados } \\
\text { se encuentra en forma detallada, } \\
\text { proporcionando datos detallados del total del } \\
\text { género femenino y masculino y el total de } \\
\text { ambos. }\end{array}$ & $\begin{array}{l}\text { Nivel 1. } \\
\text { Lectura literal }\end{array}$ & $\begin{array}{l}\text { La respuesta del estudiante presenta las } \\
\text { palabras 'evolución de titulados' y } \\
\text { 'género femenino y masculino', lo que } \\
\text { nos da indicios que observa el título y la } \\
\text { variable. }\end{array}$ \\
\hline $\begin{array}{l}\text { Tarea B, estudiante } 7 \text { : } \\
\text { Del año } 2012 \text { hasta el año } 2016 \text { la cantidad } \\
\text { de mujeres que se han titulado de carreras } \\
\text { de pregrado ha aumentado. } \\
\text { La cantidad de hombres que se han titulado }\end{array}$ & $\begin{array}{l}\text { Nivel } 1 . \\
\text { Lectura literal } \\
\text { Nivel } 2 . \\
\text { Comparativo }\end{array}$ & $\begin{array}{l}\text { La respuesta del estudiante exhibe las } \\
\text { palabras 'titulado de carreras' y 'mujeres } \\
\text { - varones', lo que nos da indicios que } \\
\text { observa el título y la variable. Además, } \\
\text { presenta comparaciones por filas, al }\end{array}$ \\
\hline
\end{tabular}


no es contante (aumento y disminución)

El total de titulados ha aumentado, pero no se forma constante.

Entre 2012 y 2015 los varones titulados aumentaron, en el año 2016 disminuyó.

Tarea B, estudiante 24

En el género femenino aumento desde el año 2012 al 2018. Entre el año 2012 al 2013 entre los dos géneros en total aumentó 140.

Cada año va aumentando en ambos género femenino y masculino un cierto porcentaje solo que en el último año aumentó $1 \%$ con diferencia a los años anteriores tuvo un mayor aumento.

\section{Tarea B, estudiante 5: \\ El género femenino tiene una tendencia a} aumentar la cantidad de titulados a medida que van pasando los años, podríamos incluso decir que el año 2017 será mayor a 1067 tituladas.

Los titulados por año siempre es mayor el género femenino, que el masculino.

Entre el año 2015 y 2016 existe un retroceso en cuanto a la cantidad de titulados masculino

Entre el año 2012 y 2016 existe una tendencia a salir cada vez más titulados, aunque el aumento sea pequeño (2015-2016) siempre incrementa.

\section{Tarea B, estudiante 35}

Se rescata una tendencia del aumento de egresados con el paso de los años, así como una mayor cantidad de egresados del sexo femenino que del masculino.

También es posible observar un aumento de la ratio "titulados femeninos": "titulados masculinos" con el paso de los años, aumentando de $6 \%$ del total la diferencia a un $16,3 \%$ en el 2016 .

La tabla resulta algo ambigua, debido a que es necesario inferir que los números indican el $n^{\circ}$ de egresados (no se especifica la "evolución" a la que se refiere la tabla)

\section{Los datos sugieren que la diferencia} seguirá aumentando

La superioridad del $n^{\circ}$ de egresados femeninos se puede deber a que: son más las carreras en las que suele ingresar una mayor cantidad de mujeres, que es mayor el cupo de carreras frecuentadas por mujeres o que las mujeres muestran una mayor tendencia a titularse. indicar expresiones como 'la cantidad de mujeres que se han titulado de carreras de pregrado ha aumentado', es decir, toma como base la variable 'tiempo'.

\section{Nivel 1. La respuesta del estudiante presenta}

Lectura literal palabras ligadas directamente al valor de la variable, 'femenino - masculino';

Nivel 2.

Comparativo comparaciones de los datos por filas, al indicar palabras como 'aumento'; sin

(Inadecuado) embargo, en esta comparación presenta una falencia al indicar 'en el último año aumentó 1\%'.

$\begin{array}{ll}\begin{array}{l}\text { Nivel 1. } \\ \text { Lectura literal }\end{array} & \begin{array}{l}\text { La respuesta del estudiante exhibe palabras } \\ \text { alusivas al título y la variable; además, lee } \\ \text { frecuencias de los valores de la variable }\end{array} \\ \text { Nivel 2. } & \begin{array}{l}\text { para realizar comparaciones: 'Los } \\ \text { titulados por año siempre es mayor el } \\ \text { género femenino, que el masculino'. }\end{array} \\ \text { Nivel 3. } & \begin{array}{l}\text { Además, presenta predicciones sobre la } \\ \text { tendencia de los datos: 'existe una } \\ \text { Predictivo }\end{array} \\ & \begin{array}{l}\text { tendencia a salir cada vez más titulados... } \\ \text { siempre incrementa'. }\end{array}\end{array}$

\begin{tabular}{|c|c|}
\hline $\begin{array}{l}\text { Nivel } 1 . \\
\text { Lectura literal }\end{array}$ & $\begin{array}{l}\text { La respuesta del estudiante presenta } \\
\text { palabras alusivas al título y la variable; } \\
\text { comparaciones por filas: 'es posible }\end{array}$ \\
\hline Nivel 2. & observar un aumento; y una predicción \\
\hline Comparativo & $\begin{array}{l}\text { acerca de la tendencia de los datos: 'Los } \\
\text { datos sugieren que la diferencia seguirá }\end{array}$ \\
\hline Nivel 3. & aumentando'. Aunado a lo anterior, la \\
\hline Predictivo & $\begin{array}{l}\text { respuesta muestra una integración con el } \\
\text { contexto de manera crítica al señalar: ' } \boldsymbol{L} \boldsymbol{a}\end{array}$ \\
\hline Nivel 4. & tabla resulta algo ambigua, debido a que \\
\hline Integrativo & $\begin{array}{l}\text { es necesario inferir que los números } \\
\text { indican el } n^{\circ} \text { de egresados (no se } \\
\text { especifica la "evolución" a la que se } \\
\text { refiere la tabla)'. }\end{array}$ \\
\hline
\end{tabular}

Fuente: Elaborada por los autores

En la Tabla 4 se presentan las frecuencias de las respuestas clasificadas por nivel de comprensión alcanzado en cada tarea del estudio, y se muestran el índice del nivel ponderado 
por grupo. Este se determina asignando los valores $0,1,2,3$ y 4 respectivamente a cada nivel de comprensión y calculando la media ponderada de las frecuencias.

Tabla 4: Clasificación por nivel de comprensión alcanzado

\begin{tabular}{|c|c|c|c|c|c|c|}
\hline \multirow{2}{*}{$\begin{array}{l}\text { Lectura e } \\
\text { interpretación }\end{array}$} & \multicolumn{5}{|c|}{ Nivel de comprensión alcanzado - Grupo 1 (México) } & \multirow{2}{*}{$\begin{array}{c}\text { Índice de nivel } \\
\text { ponderado }\end{array}$} \\
\hline & Nivel 0 & Nivel 1 & Nivel 2 & Nivel 3 & Nivel 4 & \\
\hline Adecuada & 4 & 0 & 6 & 2 & 11 & \\
\hline Inadecuada & 0 & 0 & 13 & 0 & 0 & 2,44 \\
\hline Total & 4 & 0 & 19 & 2 & 11 & \\
\hline \multirow{2}{*}{$\begin{array}{l}\text { Lectura e } \\
\text { interpretación }\end{array}$} & \multicolumn{5}{|c|}{ Nivel de comprensión alcanzado - Grupo 2 (Chile) } & Índice de nivel \\
\hline & Nivel 0 & Nivel 1 & Nivel 2 & Nivel 3 & Nivel 4 & ponderado \\
\hline Adecuada & 0 & 1 & 10 & 5 & 11 & \\
\hline Inadecuada & 0 & 0 & 5 & 3 & 0 & 2,83 \\
\hline Total & 0 & 1 & 15 & 8 & 11 & \\
\hline
\end{tabular}

Fuente: Elaborada por los autores

Analizando los niveles alcanzados, se observa que la mayor proporción de las respuestas de los estudiantes se clasifican en el nivel 2 (19/36 y 15/35, México y Chile, respectivamente) y en el nivel 4 (11/36 y 11/35, México y Chile, respectivamente). En general podemos establecer que la comprensión de la información de tabla se enfocó en la comparación de datos; no obstante, la muestra de los estudiantes universitarios de México presentó más falencias en este proceso.

Los niveles de 1 al 4 poseen diversas características que ayudan a definir con más detalle los aspectos que consideran los estudiantes en la comprensión de la información. Por ello, en la Tabla 5 se exhibe un análisis más detallado de los aspectos o elementos característicos presentes en las respuestas de los estudiantes. Cabe destacar que el total de frecuencias de las respuestas clasificadas por nivel en la Tabla 5 no corresponde al mostrado en la Tabla 4, ya que al analizarlas y clasificarlas, estas presentaban elementos característicos no sólo de un nivel, sino que en su mayoría, de dos o más niveles; como se mostró en la clasificación de las respuestas en la Tabla 3.

Tabla 5: Clasificación de acuerdo al nivel de lectura y aspecto/elemento característico

\begin{tabular}{llcc}
\hline Nivel de comprensión & Aspecto o elemento característico & Grupo 1 (México) & Grupo 2 (Chile) \\
\hline \multirow{4}{*}{ Nivel 1. Lectura literal } & Variable & 0 & 0 \\
& Título y frecuencia & 0 & 0 \\
& Variable y título & 0 & 0 \\
& Variable y frecuencia & 0 & 2 \\
& Variable, frecuencia y título & $\mathbf{3 1}$ & $\mathbf{3 0}$ \\
\hline \multirow{3}{*}{ Nivel 2. Comparativo } & Manera por columnas & $\mathbf{2 2}$ & 1 \\
& Manera por filas & 0 & 11 \\
& Ambas maneras & 9 & $\mathbf{2 2}$ \\
\hline \multirow{3}{*}{ Nivel 3. Predictivo } & Predicción de tendencia & 1 & $\mathbf{1 1}$ \\
& Predicción de valor & 1 & 0 \\
\hline
\end{tabular}




\begin{tabular}{llcc}
\hline & Racional/literal & 0 & 1 \\
Nivel 4. Integrativo & Hipotético & $\mathbf{1 1}$ & $\mathbf{7}$ \\
& Crítico & 0 & 1 \\
& Hipotético y crítico & 0 & 2 \\
\hline
\end{tabular}

Fuente: Elaborada por los autores

Al realizar el análisis de los aspectos/elementos característicos presentes en las respuestas y que definen categorías en cada uno de los niveles, podemos señalar que:

1. los estudiantes de ambos países (31/36 y 30/35, México y Chile, respectivamente) que alcanzan o transitan por el nivel 1, se enfocan en la lectura literal de la(s) variable(s), su(s) frecuencia(s) y el título de la tabla de manera conjunta.

2. los estudiantes mexicanos (22/36) que alcanzan o transitan por el nivel 2 , tienden a comparar los datos por columnas identificando un aumento o decremento en los porcentajes; en cambio, los estudiantes chilenos (22/35) efectúan la comparación por ambas maneras, filas y columnas, al identificar el valor de la variable con mayor frecuencia y detectar incrementos/decrementos.

3. los estudiantes chilenos (11/36) que alcanzan o transitan por el nivel 3 tienden a dar predicciones sobre tendencias del comportamiento de los datos; mientras que sólo dos estudiantes mexicanos (2/36) establecen predicciones sobre tendencias o valores, uno en cada caso.

4. los estudiantes de ambos países (11/36 y 7/35, México y Chile, respectivamente) que alcanzan el nivel 4, realizan una integración con el contexto de manera hipotética al aceptar y evaluar la información formando hipótesis explicativas.

A partir de lo anterior, y de manera general, podemos considerar que la forma en que es presentada la información en la tabla no influye en los niveles de comprensión alcanzados por los estudiantes; ya que ambos grupos logran realizar comparaciones, predicciones y elaborar hipótesis acerca de los datos. Por otro lado, estos resultados nos generan indicios de que la formación académica influye en el tipo de elementos característicos que recupera cada grupo al realizar la lectura e interpretación de datos estadísticos, lo cual, deja abierta una línea de investigación a futuro.

\section{Conclusiones}

En respuesta a las preguntas de investigación, la mayor proporción de los estudiantes 
universitarios de ambos países alcanzan el nivel 2, algo que se ve reflejado en el índice del nivel ponderado de 2,44 y 2,83 en el grupo de México y Chile, respectivamente. Adjudicamos esta diferencia a la presencia del nivel 0 y la relativa ausencia del nivel 3 en el grupo mexicano, lo que contrasta con la ausencia de nivel 0 y presencia notoria del nivel 3 en el grupo chileno, quienes claramente estuvieron más dispuestos a vaticinar lo que ocurriría más allá de los datos expuestos en la tabla. Además, cabe señalar que alrededor del $31 \%$ de los estudiantes de ambos grupos llega al nivel 4; por lo que fueron capaces de extraer conclusiones basadas en hipótesis explicativas de la información asociada a su conocimiento del contexto permanente y/o contingente en el que se encuentran, indistintamente de las particularidades que se caracterizan en su entorno.

Por otro lado, es importante mencionar que se presentaron comprensiones inadecuadas de la información estadística en ambos grupos. En el caso chileno, el 22.8\% de las respuestas de los estudiantes presentaron errores en las características del nivel 2 y 3 , referentes a la comparación por filas y predicción de datos; es decir, identifican aumentos/decrementos y/o realizan cálculos de suma/resta, pero de manera inadecuada. En contraste, el caso mexicano presentó más falencias (36.1\%) en la comparación de datos, ya sea por filas o columnas. Por lo anterior, podemos señalar que los estudiantes universitarios, independiente del contexto, presentan comprensiones inadecuadas referentes al nivel 2. Además, en este estudio se pueden apreciar los aspectos o elementos característicos que consideran los estudiantes universitarios cuando realizan la lectura e interpretación de una tabla estadística (ver Tabla 5).

Como una línea futura, se propone llevar a cabo nuevas pesquisas analizando los niveles de comprensión de tablas y gráficas estadísticas de estudiantes de diversos niveles educativos, con el objetivo de fortalecer la jerarquía propuesta en este estudio, y con ello, establecer más a detalle la interrelación entre los niveles de lectura de Curcio y cols. (Curcio, 1989; Friel et al., 2001) y los niveles de interpretación de Aoyama (2007).

\section{Referencias}

Amorim, N. y Silva, R. (2016). Apresentação e utilização de tabelas em livros didáticos de matemática do $4^{\circ}$ e $5^{\circ}$ anos do ensino fundamental. EM TEIA - Revista de Educação Matemática e Tecnológica Iberoamericana, 7(1), 1-21.

Aoyama, K. (2007). Investigating a hierarchy of students' interpretations of graphs. International Electronic Journal of Mathematics Education, 2(3), 298-318. 
Arredondo, E-H., García-García, J.I. y López, C. (2019). Niveles de lectura de estudiantes de licenciatura: el caso de una tabla y una gráfica de líneas. Revista digital Matemática, Educación e Internet, 19(2), 1-13.

Arteaga, P., Batanero, C., Cañadas, G. y Contreras, J. M. (2011). Las tablas y gráficos estadísticos como objetos culturales. Números, 76, 55-67.

Batanero, C. (2001). Didáctica de la Estadística. Granada: Universidad de Granada.

Batanero, C., Díaz-Levicoy, D. y Arteaga, P. (2018). Evaluación del nivel de lectura y la traducción de pictogramas por estudiantes chilenos. Avances de Investigación en Educación Matemática, 14, 49-65.

Beltrão, T. M. S. (2012). Uma análise da transposição didática externa com base no que propõem documentos oficiais para o ensino de gráficos estatísticos. Revista Paranaense de Educação Matemática, 1(1), 131-152.

Bivar, D. y Selva, A. (2011). Analisando atividades envolvendo gráficos e tabelas nos livros didáticos de matemática. En R. Borba, C. Monteiro y A. Ruiz (Eds.), Anais do XIII Conferência Interamericana de Educação Matemática (pp. 1-12). Recife: Universidad Federal de Pernambuco.

Cabral dos Santos, K.B. y Selva, A.C.V. (2011). Interpretação de gráficos: explorando a concepção de professores. En R. Borba, C. Monteiro y A. Ruiz (Eds.), Anais do XIII Conferência Interamericana de Educação Matemática (pp. 1-11). Recife: Universidad Federal de Pernambuco.

Carmona, D. y Cruz, D. (2016). Niveles de comprensión de la información contenida en tablas y gráficas estadísticas: un estudio desde la jerarquía de Kazuhiro Aoyama (Tesis de Maestría). Universidad de Medellín, Colombia.

Curcio, F. R. (1989). Developing graph comprehension. Reston, VA: NCTM.

Del Pino, G. y Estrella, S. (2012). Educación estadística: Relaciones con la matemática. Pensamiento Educativo. Revista de Investigación Educacional Latinoamericana, 49(1), 53-64.

Díaz-Levicoy, D., Batanero, C., Arteaga, P. y López-Martín, M. (2015). Análisis de los gráficos estadísticos presentados en libros de texto de educación primaria chilena. Educaçao Matematica Pesquisa, 17(4), 715-739.

Díaz-Levicoy, D., Morales, R. y López-Martín, M. (2015). Tablas estadísticas en los libros de texto chilenos de $1^{\circ}$ y $2^{\circ}$ año de Educación Primaria. Revista Paranaense de Educação Matemática, 4(7), 10-39.

Díaz-Levicoy, D., Sepúlveda, A., Vásquez, C. y Opazo, M. (2016). Lectura de tablas estadísticas por futuras maestras de Educación Infantil. Educação Matemática Pesquisa, 18(3), 1099-1115.

Espinoza, N. (2015). Tablas y gráficos de barras a través del ciclo del pensamiento estadístico. Un estudio con alumnos de primer grado de Educación Primaria (Tesis de Magíster). Pontificia Universidad Católica del Perú, Perú. 
Estrella, S. (2014). El formato tabular: una revisión de literatura. Revista Actualidades Investigativas en Educación, 14(2), 1-23.

Estrella, S. y Olfos, R. (2012). La taxonomía de comprensión gráfica de Curcio a través del gráfico de Minard: una clase en séptimo grado. Educación Matemática, 24(2), 123-133.

Estrella, S. y Olfos, R. (2015). Transnumeración de los datos: el caso de las tablas de frecuencia. En P. Scott y A. Ruíz (Eds.), XIV Conferencia Interamericana de Educación Matemática (pp. 1-7). Chiapas: Universidad del Valle de México.

Eudave, D. (2009). Niveles de comprensión de información y gráficas estadísticas en estudiantes de centros de educación básica para jóvenes y adultos de México. Educación Matemática, 21(2), 5-37.

Friel, S., Curcio, F. y Bright, G. (2001). Making sense of graphs: critical factors influencing comprehension and instructional implications. Journal for Research in mathematics Education, 32 (2), 124-158.

Fuentes, S., Arteaga, P. y Batanero, C. (2014). Gráficos estadísticos y tablas: una actividad exploratoria en Educación Infantil. En F. España (Ed.), Actas del XV Congreso de Enseñanza y Aprendizaje de las Matemáticas (pp. 385-393). Baeza: Sociedad Andaluza de Educación Matemática THALES.

Gabucio, F., Martí, E., Enfedaque, J., Gilabert, S. y Konstantinidou, A. (2010). Niveles de comprensión de las tablas en alumnos de primaria y secundaria. Cultura $y$ Educación, 22(2), 183-197.

Gal, I. (2002). Adult's statistical literacy: Meaning, components, responsibilities. International Statistical Review, 70(1), 1-25.

Gal, I. y Murray, S. T. (2011). Responding to diversity in users' statistical literacy and information needs: Institutional and educational implications. Statistical Journal of the International Association for Official Statistics, 27(3-4), 185-195.

Gea, M., Arteaga, P. y Cañadas, G. (2017). Interpretación de gráficos estadísticos por futuros profesores de Educación Secundaria. Avances de Investigación en Educación Matemática, $12,19-37$.

Guimarães, G., Gitirana, V., Cavalcanti, M. y Marques, M. (2008). Análise das atividades sobre representações gráficas nos livros didáticos de matemática. En V. Gitirana, F. Bellemain y V. Andrade (Eds.), Anais do $2^{\circ}$ Simpósio Internacional de Pesquisa em Educação Matemática (pp. 1-12). Recife: Universidad Federal de Pernambuco.

Ochoa, E. (2015). El ciclo de investigación del pensamiento estadístico relacionado con tablas de doble entrada. Un estudio con alumnos del segundo grado de Educación Primaria (Tesis de Magíster). Pontificia Universidad Católica del Perú, Perú.

Pagan, A., Leite, A. P., Magina, S. y Cazorla, I. (2008). A leitura e interpretação de gráficos e tabelas no Ensino Fundamental e Médio. En V. Gitirana, F. Bellemain y V. Andrade (Eds.), Anais do $2^{\circ}$ Simpósio Internacional de Pesquisa em Educação Matemática (pp. 1-10). Recife: Universidad Federal de Pernamburgo. 
Pereira, E. L. y Conti, K. C. (2012). Interpretando tabelas e construindo gráficos com alunos do $3^{\circ}$ ano do Ensino Fundamental. En Tommasiello, M. et al. (Ed.). Didática e Práticas de Ensino na Realidade Escolar Contemporânea: constatações, análises e proposições (pp. 5294-5302). Araraquara: Junqueira \& Marin.

Ridgway, J., Nicholson, J. y McCusker, S. (2008). Mapping new statistical literacies and illiteracies. En International Conference on Mathematics Education, Trabajo presentado en el 11th International Congress on Mathematics Education. Monterrey, Mexico.

Rodríguez, F. y Sandoval, P. (2012). Habilidades de codificación y descodificación de tablas y gráficos estadísticos: un estudio comparativo en profesores y alumnos de pedagogía en enseñanza básica. Avaliação: Revista de la avaliação da Educação Superior, 17(1), 207235.

Sepúlveda, A., Díaz-Levicoy, D. y Jara, D. (2018). Evaluación de la comprensión sobre Tablas Estadísticas en estudiantes de Educación Primaria. Bolema, 32(62), p. 869-886.

Silva, M. y Guimarães, G. (2013). O conceito de escala em livros didáticos de matemática do $4^{\circ}$ e $5^{\circ}$ ano do ensino fundamental. Anais do XI Encontro Nacional de Educação Matemática (pp. 1-14). Curitiba: Pontificia Universidad Católica de Paraná.

Universidad de Los Lagos. (2016). Anuario Estadístico Institucional 2016. Recuperado de http://www.ulagos.cl/wp-content/uploads/2018/07/ANUARIO-ESTADISTICOINSTITUCIONAL-2016-UNIVERSIDAD-DE-LOS-LAGOS.pdf

Recebido em: 30/03/2019

Aceito em: 27/06/2019 